\title{
A rare case of miliary tuberculosis accompanying perihepatitis
}

eISSN: 2383-4625

\author{
Ou Jun Kwon', Suk Woo Lee ${ }^{1,2}$, Mun Sun Jang ${ }^{1,3}$, Sang Chul Kim', \\ Ji Han Lee', Hoon Kim ${ }^{1,2}$ \\ 'Department of Emergency Medicine, Chungbuk National University Hospital, Cheongju, Korea \\ ${ }^{2}$ Department of Emergency Medicine, Chungbuk National University College of Medicine, Cheongju, Korea \\ ${ }^{3}$ Department of Emergency Medical Technology, Chungbuk Health \& Science University, Cheongju, Korea
}

Perihepatic capsulitis is associated with various diseases, such as Fitz-Hugh-Curtis syndrome, systemic lupus erythematosus, perforated cholecystitis, perforated hepatic abscess, and tuberculous peritonitis. Miliary tuberculosis is present in about 2\% of all reported cases of tuberculosis and is characterized by the widespread millet-like hematogenous dissemination of Mycobacterium tuberculosis. We describe a 24-year-old virgin patient presenting with right upper quadrant and costovertebral angle pain. Diffuse perihepatic capsular enhancement was observed in abdominal computed tomography scans. Chest radiography showed miliary tuberculosis, and a polymerase chain reaction hybridization assay of sputum revealed the presence of $M$. tuberculosis. Symptoms improved after administering anti-tuberculosis medications. This report describes a rare case of miliary tuberculosis accompanying perihepatitis.

Keywords Perihepatitis; Tuberculosis, military; Mycobacterium tuberculosis

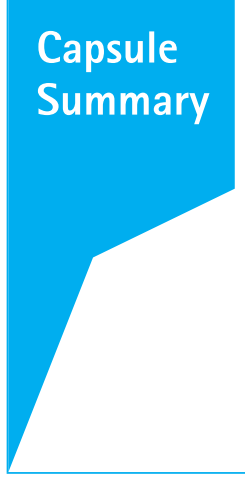

What is already known

Perihepatitis is associated with various diseases such as Fitz-Hugh-Curtis syndrome, systemic lupus erythematosus, perforated cholecystitis, perforated hepatic abscess, and tuberculous peritonitis.

What is new in the current study

Perihepatitis associated with miliary tuberculosis is very rare.
Received: 15 March 2018

Revised: 19 April 2018

Accepted: 26 April 2018

Correspondence to: Hoon Kim Department of Emergency Medicine, Chungbuk National University College of Medicine, 1 Chungdae-ro, Seowongu, Cheongju 28644, Korea

E-mail: nichekh2000@chungbuk.ac.kr ORCID

http://orcid.org/0000-0002-2133-0311

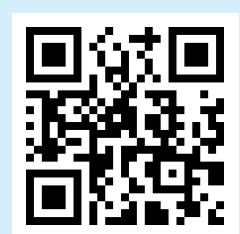

How to cite this article:

Kwon OJ, Lee SW, Jang MS, Kim SC, Lee $\mathrm{JH}, \mathrm{Kim} \mathrm{H}$. A rare case of miliary tuberculosis accompanying perihepatitis. Clin Exp Emerg Med 2019;6(3):264-267.

This is an Open Access article distributed under the terms of the Creative Commons Attribution Non-Commercial License (http:// creativecommons.org/licenses/by-nc/4.0/). 


\section{INTRODUCTION}

Perihepatitis (hepatic capsular enhancement) is defined as inflammation of the peritoneal capsule of the liver and is associated with various diseases, such as Fitz-Hugh-Curtis syndrome, systemic lupus erythematosus, perforated cholecystitis, perforated hepatic abscess, and tuberculous peritonitis. ${ }^{1-3}$ Mycobacterium tuberculosis is a gram-positive bacillus that usually causes pulmonary infection, as well as extra-pulmonary infection. Miliary tuberculosis refers to clinical disease resulting from hematogenous dissemination of $M$. tuberculosis. Perihepatitis associated with M. tuberculosis is rare, such as in cases of genital tuberculosis and peritoneal tuberculosis. ${ }^{4,5}$ We encountered a rare case of miliary tuberculosis accompanying perihepatitis in a 24-year-old woman without sexual experience, who visited the emergency department with the chief complaint of right upper quadrant (RUQ) pain.

\section{CASE REPORT}

A 24-year-old virgin woman presented to the emergency department with a 3-day history of RUQ abdominal pain, which worsened with walking, running, and deep breathing. There were no other gastrointestinal symptoms, such as vomiting or diarrhea. The patient also complained of sweating at night, cough, sputum, and weight loss of $10 \mathrm{~kg}$ in the previous month. Her sister had developed pulmonary tuberculosis one year ago.

On physical examination, RUQ tenderness was obvious, with right costovertebral angle tenderness and a positive Murphy's sign. We auscultated coarse breath sounds throughout both lungs. Vital signs on admission were as follows: blood pressure, 100/60 $\mathrm{mmHg}$; pulse rate, 100 beats/min; respiratory rate, 24 breaths/min; and body temperature, $38.6^{\circ} \mathrm{C}$. Blood tests showed a leukocyte count of $10,200 / \mu \mathrm{L}$ (reference; 4,000 to $10,000 / \mu \mathrm{L}$ ) and C-reactive protein level of $11.20 \mathrm{mg} / \mathrm{dL}$ (reference, $<0.3 \mathrm{mg} / \mathrm{dL}$ ). Acidfast bacillus culture and polymerase chain reaction hybridization
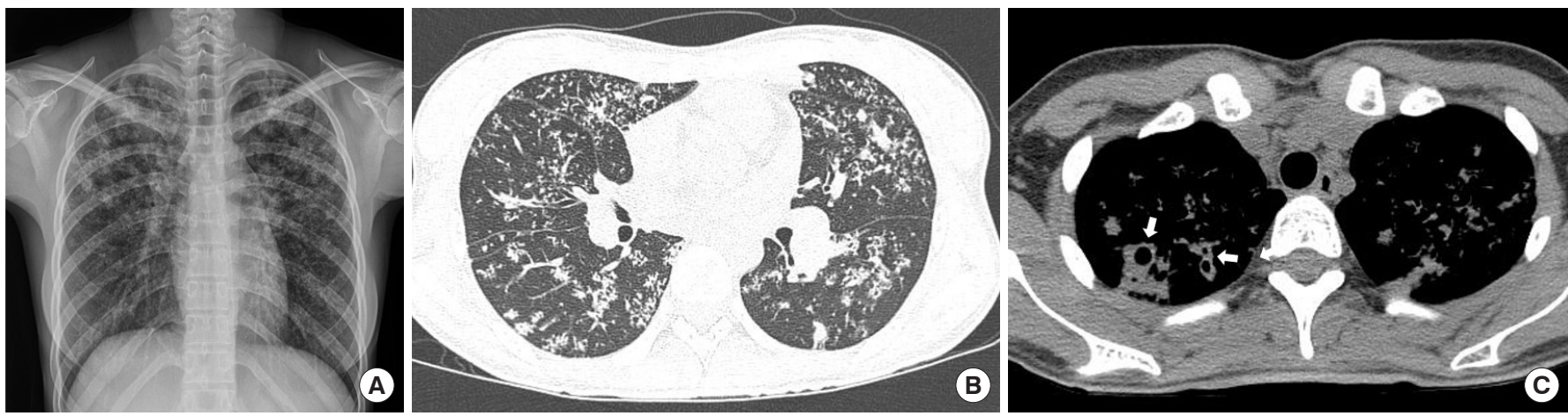

Fig. 1. Chest radiographic findings. (A) Chest posteroanterior view shows widely spread, small, patchy, and nodular opacities throughout both lung parenchyma. ( $\mathrm{B}, \mathrm{C})$ Computed tomography images of the chest show cavitary nodules (arrows), centrilobular nodules, branching structures, and consolidation in both lung parenchyma.
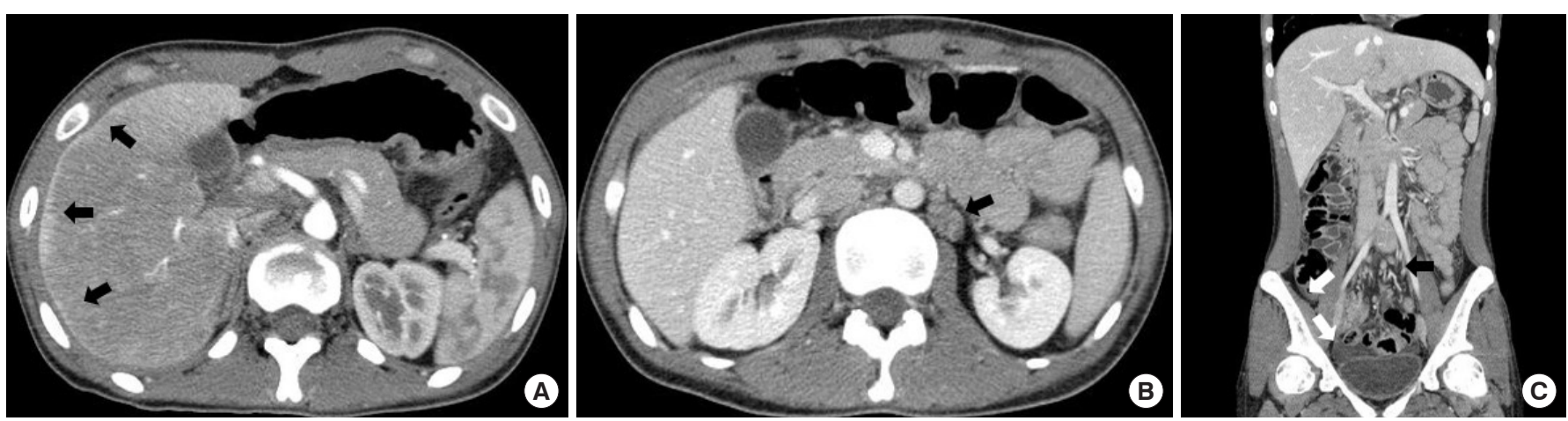

Fig. 2. Computed tomography of the abdomen. (A, B) Axial views reveal a thickened linear capsular enhancement of the liver on an arterial-phase image, enlarged paraaortic lymph nodes with internal necrosis, and small bowel mesentery (black arrows). (C) A coronal view shows a moderate amount of ascites in the pelvic and peritoneal cavities (white arrows). 
assay of the sputum revealed the presence of $M$. tuberculosis. Chest radiography showed widely spread small nodular opacities throughout both lung parenchyma (Fig. 1A). Computed tomography (CT) of the chest showed cavitary nodules, centrilobular nodules, branching structures, and consolidation in both lung parenchyma (Fig. 1B, C). Dynamic contrast-enhanced CT of the abdomen revealed diffuse perihepatic capsular enhancement of the liver (Fig. 2A), enlarged paraaortic lymph nodes, small bowel mesentery with internal necrosis (Fig. 2B), and a moderate amount of ascites in the pelvic and peritoneal cavities (Fig. 2C). Anti-tuberculosis therapy with oral isoniazid, rifampicin, ethambutol, pyrazinamide, and pyridoxine was initiated. Eight days after admission, the patient showed an improvement in symptoms and was discharged.

\section{DISCUSSION}

We report this case of perihepatitis that was associated with miliary tuberculosis, which occurred in a young immunocompetent woman who did not exhibit pelvic inflammatory disease symptoms.

Perihepatitis is characterized by perihepatic inflammation associated with Fitz-Hugh-Curtis syndrome (which is frequently caused by sexually transmitted microorganisms, such as Chlamydia trachomatis and Neisseria gonorrhoeae), systemic lupus erythematosus, drug side effects, and non-tuberculosis mycobacterial infection. ${ }^{6-9}$

Classical miliary tuberculosis is defined as millet-like seeding of $M$. tuberculosis in the lungs, as evidenced on chest radiographs, which also accounts for up to $20 \%$ of all extra-pulmonary tuberculosis. ${ }^{10}$ The risk of extra-pulmonary tuberculosis is increased in immunocompromised patients with cirrhosis, human immunodeficiency virus infection and acquired immune deficiency syndrome, diabetes, and cancer. Although there are a few case reports of perihepatitis associated with genital tuberculosis or peritoneal tuberculosis infected with M. tuberculosis, there have been no case reports concerning perihepatitis associated with miliary tuberculosis in the published English literature to date.

CT is the gold standard method for non-invasive diagnosis of perihepatitis, in which hepatic capsule enhancement in the arterial phase can be observed relative to other phases; this enhancement is due to an increased blood flow or inflammation of the hepatic capsule., ${ }^{1,11}$ It may also be helpful to demonstrate the 'violin string-like adhesions' observed on laparoscopic examination. Abdominal CT in our case showed enlarged multiple intra-abdominal lymphadenopathy with internal necrosis, which may demonstrate extra-pulmonary spreading of miliary tuberculosis. We pro- pose three potential mechanisms for the pathway of miliary tuberculosis to the subphrenic or perihepatic region, as follows: 1) transphrenic descending spread of $M$. tuberculosis from the lungs, 2) hematogenous dissemination, and 3) translymphatic spread.

We presented a rare case of perihepatitis associated with miliary tuberculosis, which occurred in a 24-year-old virgin woman with the chief complaint of RUQ abdominal pain. This illustrates that emergency physicians should include miliary tuberculosis in the differential diagnosis of perihepatitis.

\section{CONFLICT OF INTEREST}

No potential conflict of interest relevant to this article was reported.

\section{ACKNOWLEDGMENTS}

This research was supported by the Basic Science Research Program through the National Research Foundation of Korea funded by the Ministry of Education, Science and Technology (2015R1A2 A2A01002303 and NRF-2016R1D1A1B04930247).

\section{REFERENCES}

1. Kim S, Kim TU, Lee JW, et al. The perihepatic space: comprehensive anatomy and CT features of pathologic conditions. Radiographics 2007;27:129-43.

2. Sonavane $A D$, Rathi PM. Fitz-Hugh-Curtis syndrome. Indian J Med Res 2017;145:147.

3. Laabidi J, Ajili F. Perihepatitis in systemic lupus erythematosus. Pan Afr Med J 2015;20:280.

4. Sharma JB, Roy KK, Gupta N, Jain SK, Malhotra N, Mittal S. High prevalence of Fitz-Hugh-Curtis syndrome in genital tuberculosis. Int J Gynaecol Obstet 2007;99:62-3.

5. Hong DG, Choi MH, Chong GO, et al. Fitz-Hugh-Curtis syndrome: single centre experiences. J Obstet Gynaecol 2010;30: 277-80.

6. Centers for Disease Control and Prevention (CDC). Update to CDC's sexually transmitted diseases treatment guidelines, 2010: oral cephalosporins no longer a recommended treatment for gonococcal infections. MMWR Morb Mortal Wkly Rep 2012 61:590-4.

7. Schoenwaelder M, Stuckey SL. Perihepatitis associated with systemic lupus erythematosus: computed tomography findings. Australas Radiol 2005;49:179-81.

8. Tsukada K, Teruya K, Tasato D, Gatanaga H, Kikuchi Y, Oka S. Raltegravir-associated perihepatitis and peritonitis: a single 
Ou Jun Kwon, et al.

case report. AIDS 2010;24:160-1.

9. Jang HY, Burbelo PD, Chae YS, Kim T, Cho Y, Park HT. Nontuberculous mycobacterial infection in a clinical presentation of Fitz-Hugh-Curtis syndrome: a case report with multigene diagnostic approach. BMC Womens Health 2014;14:95.

10. Blumberg HM, Burman WJ, Chaisson RE, et al. American Tho- racic Society/Centers for Disease Control and Prevention/Infectious Diseases Society of America: treatment of tuberculosis. Am J Respir Crit Care Med 2003;167:603-62.

11. Joo SH, Kim MJ, Lim JS, Kim JH, Kim KW. CT diagnosis of FitzHugh and Curtis syndrome: value of the arterial phase scan. Korean J Radiol 2007;8:40-7. 